\section{DIAGNOSTIC AND PROGNOSTIC UTILITY OF CARDIOVASCULAR MAGNETIC RESONANCE IMAGING IN HEART FAILURE WITH PRESERVED EJECTION FRACTION}

1,*P Kanagala, ${ }^{2}$ ASH Cheng, ${ }^{1} \mathrm{~J}$ McAdam, ${ }^{1} \mathrm{AM}$ Marsh, ${ }^{1} \mathrm{IB}$ Squire, ${ }^{1} \mathrm{LL}$ Ng, ${ }^{1} \mathrm{GP}$ McCann. ${ }^{1}$ Department of Cardiovascular Sciences and NIHR Cardiovascular Biomedical Research Unit, Glenfield Hospital, Leicester, UK; ${ }^{2}$ Kettering General Hospital, Kettering and NIHR Cardiovascular Biomedical Research Unit, Glenfield Hospital, Leicester, UK

\subsection{6/heartjnl-2016-309668.12}

Purpose Heart failure with preserved ejection fraction (HFpEF) is a poorly characterised condition. We aimed to phenotype patients with HFpEF using multiparametric stress cardiovascular magnetic resonance imaging (CMR) and assess the relationship to clinical outcomes.

Methods and Results Patients were recruited as part of an observational, single-centre, cohort study. Inclusion criteria were: clinical or radiographic evidence of heart failure (HF) and ejection fraction $>50 \%$ on transthoracic echocardiography (TTE). Exclusion criteria were: myocardial infarction (MI) in the preceding 6 months, suspected or confirmed cardiomyopathy/ constrictive pericarditis, non-cardiovascular life expectancy $<6$ months and severe valve/ lung/ renal disease.

Patients labelled as HFpEF ( $\mathrm{n}=154,51 \%$ male, mean age $72.4 \pm 10$ years) underwent TTE and CMR during a single study visit. The CMR protocol comprised cine, stress/rest perfusion and late gadolinium enhancement imaging on a 3-Tesla scanner. Follow-up outcome data (death or HF hospitalisation) was captured after a minimum of 6 months.

CMR detected previously undiagnosed pathology in 42 patients (27\%), who had similar baseline characteristics to those without a new diagnosis (see Table 1). These diagnoses consisted of: coronary artery disease $(n=20$, including 14 with 'silent' MI), microvascular dysfunction $(\mathrm{n}=11)$, probable or definite hypertrophic cardiomyopathy $(\mathrm{n}=10)$ and constrictive pericarditis $(n=5)$. Four patients had dual pathology. During followup (median $=623$ days), those patients with a new CMR diagnosis were at higher risk (see Figure 1) of adverse outcome for the composite end-point (hazard ratio log rank test: $\mathrm{p}=0.047$ ). In multivariate analysis, the 'new CMR diagnoses' group remained an independent predictor of outcome (hazard ratio: 1.92; $95 \% \mathrm{CI}: 1.07$ to $3.45 ; \mathrm{p}=0.03$ ).

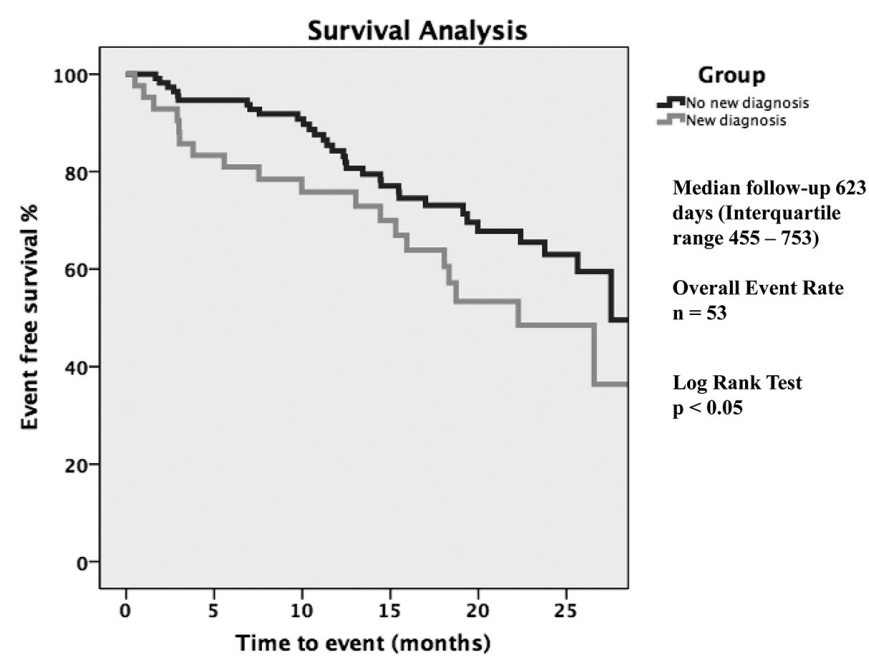

Abstract 12 Figure 1 Kaplan-Meier survival plots for the composite end-point of death and/or re-hospitalisation from heart failure stratified according to new CMR diagnosis versus no new diagnosis
Conclusion Stress CMR diagnosed new significant pathology in $27 \%$ of patients with HFpEF and these patients were at increased risk of death and HF hospitalisation.

Conflicts of interest On behalf of all authors, there are no conflicts of interest to declare.

Abstract 12 Table 1 Baseline characteristics of the study cohort who underwent $\mathrm{CMR}$

\begin{tabular}{|c|c|c|c|c|}
\hline & All & $\begin{array}{l}\text { No new diagnosis } \\
\text { group } \\
(\mathrm{n}=112)\end{array}$ & $\begin{array}{l}\text { New diagnosis } \\
\text { group } \\
(n=42)\end{array}$ & $\begin{array}{l}\mathrm{p} \\
\text { value }\end{array}$ \\
\hline \multicolumn{5}{|l|}{ Demographics } \\
\hline Age, years & $72 \pm 10.0$ & $72.6 \pm 9.3$ & $71.7 \pm 11.8$ & 0.61 \\
\hline Male & $78(51)$ & $54(48)$ & $24(57)$ & 0.32 \\
\hline \multicolumn{5}{|l|}{ Clinical findings } \\
\hline Atrial fibrillation & $72(47)$ & $50(45)$ & $24(52)$ & 0.42 \\
\hline Heart rate & $70.4 \pm 14.2$ & $70.0 \pm 13.6$ & $71.5 \pm 15.8$ & 0.57 \\
\hline $\begin{array}{l}\text { Systolic Blood } \\
\text { Pressure }\end{array}$ & \multicolumn{3}{|c|}{ Pressure } & 0.61 \\
\hline $\begin{array}{l}\text { Diastolic Blood } \\
\text { Pressure }\end{array}$ & $74.0 \pm 12.2$ & $74.0 \pm 11.8$ & $74.0 \pm 13.2$ & 0.99 \\
\hline $\begin{array}{l}\text { Body Mass Index } \\
\text { NYHA }\end{array}$ & \multicolumn{3}{|c|}{ NYHA } & 0.66 \\
\hline I/II & $106(69)$ & $77(69)$ & $29(69)$ & 0.97 \\
\hline III/IV & $48(31)$ & $35(31)$ & $13(31)$ & \\
\hline \multicolumn{5}{|l|}{ Medical History } \\
\hline Known CAD & $32(21)$ & - & - & - \\
\hline Hypertension & $139(90)$ & $111(89)$ & $39(93)$ & 0.60 \\
\hline Diabetes & $75(49)$ & $54(48)$ & $21(50)$ & 0.88 \\
\hline $\begin{array}{l}\text { COPD or Asthma } \\
\text { Chest radiography }\end{array}$ & \multicolumn{3}{|c|}{ Chest radiography } & 0.21 \\
\hline $\begin{array}{l}\text { Pulmonary oedema } \\
\text { Medication }\end{array}$ & \multicolumn{2}{|c|}{ Medication } & 31 (74) & 0.69 \\
\hline Aspirin & $54(35)$ & $42(38)$ & $12(29)$ & 0.30 \\
\hline Beta-blocker & $99(64)$ & $74(66)$ & $25(60)$ & 0.45 \\
\hline $\begin{array}{l}\text { ACE inhibitor or } \\
\text { ARB }\end{array}$ & $130(84)$ & $97(87)$ & $33(79)$ & 0.22 \\
\hline Statin & $97(63)$ & $70(63)$ & $27(64)$ & 0.84 \\
\hline Loop diuretic & $125(81)$ & $91(81)$ & $34(81)$ & 0.97 \\
\hline \multicolumn{5}{|l|}{ Biochemistry } \\
\hline Sodium & $139.2 \pm 3.4$ & $139.1 \pm 3.6$ & $139.6 \pm 2.6$ & 0.39 \\
\hline Urea & $8.7 \pm 3.8$ & $8.8 \pm 4.0$ & $8.3 \pm 3.5$ & 0.46 \\
\hline eGFR & $65.4 \pm 18.8$ & $66.0 \pm 18.7$ & $63.5 \pm 19.3$ & 0.46 \\
\hline BNP (median, IQR) & $\begin{array}{l}144.6(66- \\
259)\end{array}$ & $\begin{array}{l}133.6 \pm(57.5- \\
251.1)\end{array}$ & $\begin{array}{l}175.4 \pm(110.7- \\
262.9)\end{array}$ & ${ }^{\star} 0.12$ \\
\hline \multicolumn{5}{|l|}{$C M R$} \\
\hline LVEF & $57.0 \pm 6.1$ & $57.0 \pm 5.9$ & $57.0 \pm 6.5$ & 0.98 \\
\hline LVEDVI & $74.3 \pm 18.2$ & $73.3 \pm 16.9$ & $77.1 \pm 21.4$ & 0.26 \\
\hline LVESVI & $32.6 \pm 10.6$ & $32.1 \pm 9.6$ & $34.1 \pm 12.8$ & 0.30 \\
\hline
\end{tabular}

ACE: angiotensin converting enzyme, ARB: angiotensin II receptor blocker, BNP: B-type natriuretic peptide, CAD: significant coronary artery disease, CMR: cardiovascular magnetic resonance imaging, COPD: chronic obstructive pulmonary disease, eGFR: estimated glomerular filtration rate, LVEF: left ventricular ejection fraction, LVEDVI left ventricular end-diastolic volume indexed to body surface area, LVESVI: left ventricular end-systolic volume indexed to body surface area.

Values are mean \pm SD or $n(\%)$. The $p$ values are quoted for the independent-samples Ttest or chi-square test for continuous or categorical variables respectively.

${ }^{*} \mathrm{p}$ value refers to $\operatorname{zog}_{10}$ transformed BNP 